\title{
EFFECT OF COMPACT OBJECTS NEAR Be STARS
}

Krishna M.V. Apparao and S.P. Tarafdar Tata Institute of Fundamental Research Homi Bhabha Road, Bombay 400005, India

Several Be stars are identified with bright X-ray sources. (Rappaport and Van den Heuvel, 1982). The bright $x$-ray emission and observed periodicities indicate the existence of compact objects (white dwarfs, neutron stars or black holes) near the Be stars. A prime example is the brightest X-ray source A0538-66 in LMC, which contains a neutron star with a rotation period of $59 \mathrm{~ms}$. Apparao (1985) explained the X-ray emission, which occurs in periodic flares, by considering an inclined eccentric orbit for the neutron star around the assumed Be-star. The neutron star when it enters a gas ring (around the Be-star) accreting matter giving out $\mathrm{x}$-rays.

The $\mathrm{x}$-ray emission from the compact objects, when the gas ring from the Be-star envelopes the objects, has interesting consequences. The $\mathrm{x}-$ ray emission produces an ionized region (compact object stromgren sphere or COSS) in the gas surrounding the compact object (CO). Thus the periodic $\mathrm{H}_{\alpha}$ emission observed can be explained as the line emission from COSS in response to the observed periodic $\mathrm{X}$-ray emission (Apparao and Tarafdar, 1986). We have also calculated the nature of coss in the case of A0538-66 and is shown in Fig. 1. We have calculated the continuum emission in the ultraviolet and the optical regions and these agree (Fig. 2) with observed value. The C IV, He II ultraviolet line emission calculated also agrees with observation. The visual magnitude of light from coss is 12.5 compared to the Be star magnitude of 15 . Thus the emission from coss overwhelms the stellar emission during $\mathrm{X}$-ray emission.

In addition to COSS, the Be star itself produced an ionization region (BESS). If the line emission from BESS and coss are comparable, a $V / R$ variation is produced as shown in Fig. 3 (Apparao and Tarafdar 1986b). The CO moves around the Be star in a binary orbit and the coss emission consequently has a Doppler shift corresponding to the position in the ring with respect to the observer. The coss contribution moves across the line profile from violet end to the red end and vice versa resulting in a $V / R$ variation.

Apparao, K.M.V., Ap.J. 287, 338. Apparao, K.M.V. and S.P. Tarafdar, 1986, Astron. Astrophys., 155, 423. Apparao, K.M.V. and S.P. Tarafdar, 1986b, Astron. Astrophys. (in press) Rappaport, S. A. and Van den Heuve1, E. P. J., 1982, I.A.U. Symp. No. 98, D. Reide1 Pub1.ishing Co., Holland 
Fig. 1: The ionization states of $\mathrm{H}$, He and $C$ IV in the stromgren sphere produced by $\mathrm{X}$-rays from the neutron star in the Be star binary A0538-66 in LMC.
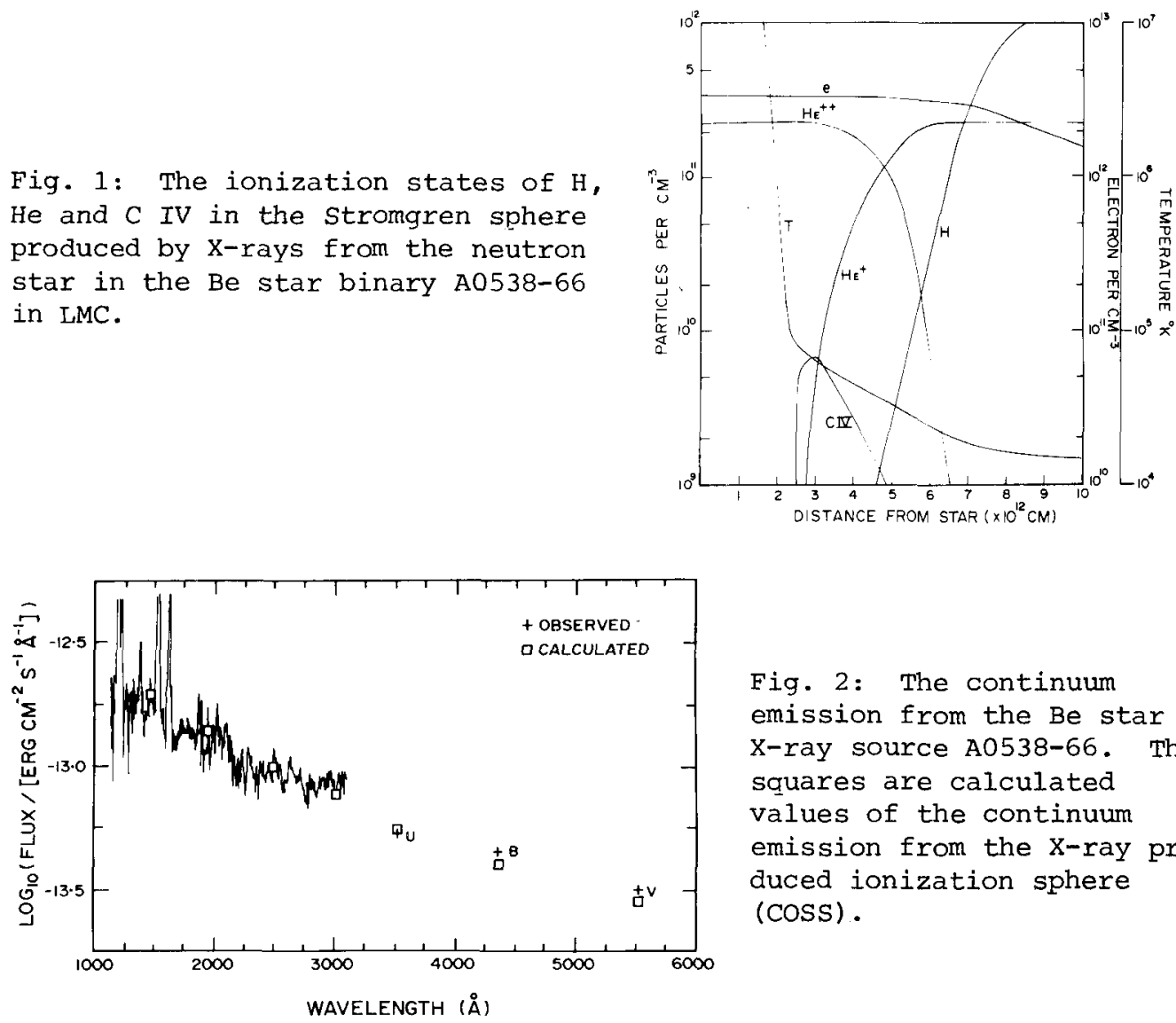

Fig. 2: The continuum emission from the Be star $\mathrm{x}$-ray source A0538-66. The squares are calculated values of the continuum emission from the $\mathrm{X}$-ray produced ionization sphere (COSS).

Fig. 3: $V / R$ variation in $\mathrm{X}-$ ray emitting Be stars binaries. The figure shows (assumed) schematic profiles from compact object ionization sphere (COSS) and $\mathrm{Be}$ star ionization sphere (BESS) (a). Their superposition is shown in (b) for different positions of the Be star and the compact object (c). The hatched portion represent ionized regions.
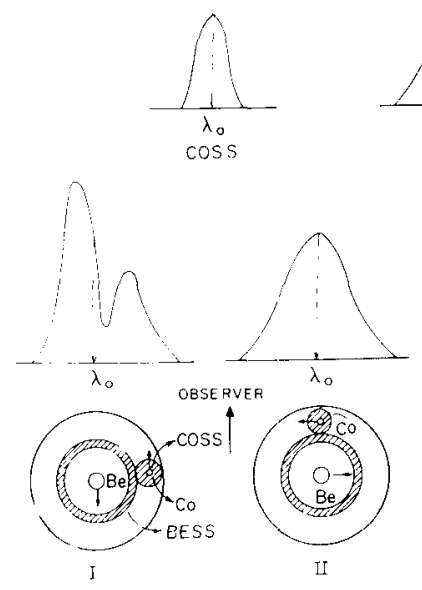

(o)

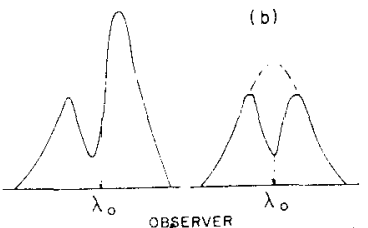

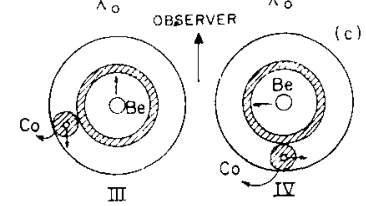




\section{DISCUSSION FOLLOWING TARAFDAR}

Henrichs:

$\mathrm{X}$-ray pulse period measurements indicated an eccentricity of the order of 0.7 . Would this alter your $V / R$ modeling?

Tarafdar:

$\mathrm{V} / \mathrm{R}$ variability will depend on the extent of the disk. If the extent is large, an eccentricity of 0.7 will not effect it, but a much larger eccentricity like $\sim 0.9$ will effect the $\mathrm{V} / \mathrm{R}$ variability provided the size is not too large. 